Cahiers « Mondes anciens »

ANCIENS

Histoire et anthropologie des mondes anciens

$11 \mid 2018$

La « civilisation » : critiques épistémologique et historique

\title{
Civilisation et Kultur : de Friedrich August Wolf à Sigmund Freud
}

Civilization and Kultur: from Friedrich August Wolf to Sigmund Freud

\section{Claude Calame}

\section{(2) OpenEdition}

Journals

Édition électronique

URL : http://journals.openedition.org/mondesanciens/2063

DOI : $10.4000 /$ mondesanciens.2063

ISSN : 2107-0199

Éditeur

UMR 8210 Anthropologie et Histoire des Mondes Antiques

Référence électronique

Claude Calame, "Civilisation et Kultur : de Friedrich August Wolf à Sigmund Freud », Cahiers " Mondes anciens » [En ligne], 11 | 2018, mis en ligne le 26 mars 2018, consulté le 19 avril 2019. URL : http:// journals.openedition.org/mondesanciens/2063 ; DOI : 10.4000/mondesanciens.2063

Ce document a été généré automatiquement le 19 avril 2019

\section{cc)}

Les Cahiers «Mondes Anciens " sont mis à disposition selon les termes de la licence Creative Commons Attribution - Pas d'Utilisation Commerciale - Pas de Modification 4.0 International. 


\title{
Civilisation et Kultur : de Friedrich August Wolf à Sigmund Freud
}

Civilization and Kultur: from Friedrich August Wolf to Sigmund Freud

\author{
Claude Calame
}

1 Dans la collection «Sagesse des mythes", une série de dessinateurs guidés par le philosophe Luc Ferry offrent aux jeunes lectrices et lecteurs francophones une nouvelle forme de mythographie. C'est désormais sous forme de bandes dessinées que sont mis en scène les grands récits héroïques grecs. Ainsi, à l'exemple de Thésée et du Minotaure, dans l'opposition du héros aux «forces du chaos », ce serait une invitation à revenir aux «fondamentaux de notre civilisation"-commente un critique ${ }^{1}$. Non seulement une " civilisation », mais la « nôtre »: retour aux origines et appropriation énonciative d'une civilisation qui ne peut, à l'évidence, que plonger ses racines dans la mythologie grecque ; c'est une mythologie grecque qui, par ailleurs, mettrait en scène le triomphe du héros civilisateur sur les forces sauvages ...

\section{Zivilisation ou Kultur? De la civilisation à la culture de l'esprit}

2 Au début du XIx siècle à Berlin, le grand historien de la littérature grecque Friedrich August Wolf fonde le Museum der Alterthumswissenschaft. Intitulé Darstellung der AltertumsWissenschaft nach Begriff, Umfang, Zweck und Werth, ce long texte d'introduction est programmatique; l'érudit y énonce les principes fondateurs d'une nouvelle «science de l'Antiquité », appelée à devenir rapidement klassiche Philologie, philologie classique. Quant à cette nouvelle science, celui qui est le père de la théorie du caractère oral de la poésie homérique déclare: "Man nennt sie bald Philologie, bald classiche Gelehrsamkeit, bald alte Literatur, bald Humanitäts-Studien, zuweilen mit einem ihnen fremden und ganz modernen Namen, schöne Wissenschaften » (1807, p. 11). Mais les Grecs et les Romains, s'empresse-t-il d'expliquer, ne sont pas les seuls à animer ce «monde ancien »; ils le partagent en effet avec les Égyptiens, les Hébreux, les Perses, « d'autres nations encore de l'Orient ». 
Ce serait néanmoins une erreur, ajoute Wolf, de mettre ces nations de l'Orient antique sur le même pied que les Grecs et les Romains! Sans doute " civilisés ", ces peuples n'auraient pas atteint la «culture » qu'offrent au contraire la Grèce et Rome. C'est que, selon un critère discriminatoire, il convient de distinguer entre la «civilité bourgeoise» ("die bürgerliche Policirung ») des uns et la " culture spirituelle » ("die Geisteskultur ») des autres. Dans cette mesure, le champ de l'Alterthum - Antiquité doit être restreint aux deux peuples policés par la culture spirituelle, c'est-à-dire les Grecs et les Romains. Au-delà de la civilisation, ils sont les représentants de la Kultur.

Dans un second temps, Wolf situe les Grecs, davantage que les Romains, à l'origine d'une relation généalogique directe avec les Allemands. Dans la préface de ce premier numéro qui est adressé à Johann Wolfgang Goethe et où le poète est désigné comme un fin connaisseur et un digne représentant de l'esprit grec ${ }^{2}$, ce rapport de filiation directe est placé sous le signe du Grand et du Beau pour affirmer: "nous Allemands, parmi les modernes, après tant de défigurations, nous nous accordons avec le plus de fidélité aux manières du chant et du discours grecs » (Wolf 1807, p. VI). Par la littérature et les arts, les Grecs et les Romains se distinguent en effet non seulement des Orientaux, parmi lesquels les Hébreux, mais surtout des Asiates et des Africains - peuples civilisés sans doute, mais sans la culture d'une littérature ("litterarisch nicht cultivirte, nur zivilisierte Völker »; Wolf 1807, p. 18). C'est pourquoi la dénomination Alterthum sera réservée aux deux peuples affinés par la culture de l'esprit (Geistescultur), l'instruction et l'art; cela à d'autant plus forte raison que Wolf affirme que toute culture repose sur une langue qui renvoie à un mode de penser et qui renforce le patrimoine des idées et la pensée d'un peuple, d'une nation. À cet égard le grec occupe une position originaire et privilégiée (cf. Cozzo 2011, p. 343-347).

5 C'est donc dans la mouvance du Romantisme allemand que le décor est planté. En contraste avec la civilisation caractérisant les autres peuples, la culture est appelée à distinguer les Grecs, les Romains et, dans la filiation, les Allemands par la littérature et les beaux-arts. Certes, quelques années auparavant, Johann Gottfried Herder avait proposé pour la culture une diffusion universelle : il en faisait le propre de l'humanité en général. Double est en effet la "genèse» de tout être humain, génétiquement par la communication, organiquement par l'assimilation de ce qui est communiqué. Et ce second processus, qui traverse toute l'existence de l'homme, est marqué par la culture ( Kultur), en référence métaphorique à la culture d'un champ, ou par l'illumination ( Aufklärung) via l'image de la lumière. Même si c'est sur un mode mineur, les peuples de Californie ou de la Terre de feu sont cultivés et illuminés : ils ont appris à fabriquer et à utiliser des arcs et des flèches; ils ont une langue et des notions, des pratiques et des arts qu'ils ont apprises: «La chaine de la culture et de la lumière s'étend en conséquence jusqu'à l'extrémité de la terre » (" die Kette der Kultur und Aufklärung reicht aber sodann bis ans Ende der Erde »; Herder 1784-91, II, p. 335 = II, livre 9, chap. 1). Indépendamment d'une civilisation qui serait celle offerte en différentes formes par les peuples primitifs que sont les Naturvölker, la culture quant à elle se définit pour Herder comme l'expression multiforme et différenciée de l'esprit (Geist) et de l'âme (Seele) d'un peuple devenu Nation. L'acquisition individuelle et collective de la culture repose sur la Bildung, sur la formation et sur l'éducation. 


\section{Premières anthropologies des cultures}

$6 \quad \mathrm{Au}$ XIX ${ }^{\mathrm{e}}$ siècle l'idée d'une culture qui se confond avec la civilisation a été reprise par l'anthropologie anglo-saxonne. Le second terme devient pratiquement synonyme du premier. Rappelons pour mémoire la fameuse définition anthropologique qu'Edward B. Tylor donne de la culture dès l'introduction de Primitive Culture (1871, p. 1, avec le commentaire de Cuche 1996, p.16-18): "Culture ou civilisation, pris dans son sens ethnologique le plus étendu, est ce tout complexe qui comprend la connaissance, les croyances, l'art, la morale, le droit, les coutumes et les autres capacités acquises par l'homme en tant que membre de la société.» C'est d'ailleurs une définition que l'anthropologue anglais précise d'emblée dans le sous-titre de l'ouvrage: Researches into the development of mythology, philosophy, religion, language, art, and custom. Même si l'ouvrage de Tylor s'inscrit dans la perspective évolutionniste de rigueur à cette époque, cette conception compréhensive des représentations et des pratiques des hommes en société ne propose ni hiérarchie entre les cultures, ni subordination entre les peuples.

7 Mais le concept plus sociologique d'une culture de l'homme impliquant la diversité des cultures des communautés humaines va s'imposer surtout dans le domaine de l'anthropologie francophone (cf. Cuche 1996, p. 22-26). On évoquera naturellement à ce propos la définition donnée implicitement et conjointement par Émile Durkheim et Marcel Mauss (1913, p. 47, dans une célèbre « note sur la notion de civilisation ») à une culture précisément conçue comme " civilisation » :

Les institutions politiques, juridiques, les phénomènes de morphologie sociale font partie de la constitution propre de chaque peuple. Au contraire, les mythes, les contes, la monnaie, le commerce, les beaux-arts, les techniques, les outils, les langues, les mots, les connaissances scientifiques, les formes et les idéaux littéraires, tout cela voyage, s'emprunte, résulte, en un mot, d'une histoire qui n'est pas celle d'une société déterminée.

8 Au-delà de l'étrange partage entre institutions propres et ce que nous classerions du côté des manifestations pratiques et symboliques (économie incluse ?) d'une culture, au-delà de l'étrange omission de ce que nous placerions sous l'étiquette de religion, au-delà de ce que Durkheim définira comme "conscience collective» faite de représentations, d'idéaux, de valeurs, de sentiments partagés, cette définition englobante de la civilisation affirme la perméabilité des cultures, dans l'histoire. Il n'y a, à vrai dire, pas de raison de soustraire à ce processus d'interaction culturelle les institutions sociales, politiques, juridiques et religieuses avec l'idéologie qui les configure et qui les légitime. Mais c'est là un autre problème.

9 Quel que soit le périmètre qu'on lui assigne, avec cette définition large, la civilisation (ou la culture) ne saurait être couplée avec l'idée de nation ou, pire encore, avec le fantasme de l'identité nationale ; de plus, à l'écart de toute hiérarchie, les cultures et la civilisation, si l'on tient à ce concept, ne peuvent être pensées que dans la diversité de communautés culturelles en constant développement interactif, à l'écart de toute origine unique, que celle-ci soit indo-européenne, sémitique, occidentale ou grecque. 


\section{La mémoire culturelle et le « Grand Partage »}

10 Est-ce à dire que notre approche de l'Antiquité échappe désormais aux définitions de la civilisation privilégiant une civilisation occidentale plongeant ses racines dans la culture gréco-romaine?

11 Comme je l'ai rappelé ailleurs (Calame 2014), à la fin du siècle dernier, l'égyptologue et historien des religions antiques Jan Assmann a reformulé le concept de "mémoire collective ». À chaque époque toute société disposerait d'un ensemble de textes, d'images et de rites qui lui permettrait de transmettre, par l'usage, une image d'elle-même. Portant en particulier sur le passé de la communauté et fondé en tradition à la fois historiographique et religieuse, ce savoir partagé serait institué en mémoire collective ; c'est sur le «sens" (Sinn) de cette mémoire collective, d'ordre culturel, que le groupe fonderait la conscience de son unité et de sa particularité (voir par exemple Assmann 1992, p. 19-25). Or, dans une contribution théorique plus récente (Assmann 2000), l'historien des religions allemand affirme que le concept de mémoire culturelle doit être assorti d'un partage entre ce qu'il dénomme, par jeu de mot interposé, Bindungsgedächtnis et ce qu'il appelle Bildungsgedächtnis; soit la "mémoire connective» d'une part, la «mémoire formative » d'autre part.

Or l'inscription d'une mémoire collective dans une tradition pose la question des moyens permettant de constituer et d'entretenir toute mémoire culturelle, d'une part dans la dimension horizontale, dans l'espace, d'autre part dans la dimension verticale, dans le temps. Parmi ces moyens d'une communication tant horizontale que verticale, l'historien des religions antiques qu'est Assmann mentionne essentiellement, comme on l'a signalé, les pratiques rituelles et les manifestations textuelles. Les premières seraient le propre des communautés qui ne connaissent pas de système graphique et dont la tradition est entièrement orale; les secondes seraient l'apanage évident des sociétés appuyant leur tradition sur un système de transcription graphique et de diffusion écrite de la parole orale. C'est dire que, par ce biais, Assmann reprend à Claude Lévi-Strauss la célèbre distinction entre sociétés froides et sociétés chaudes pour inscrire implicitement le concept de mémoire culturelle dans le "Great Divide », le «Grand Partage » mis en doute puis reproduit par Jack Goody (1977, p. 146-162). Sociétés «froides », sans histoire, les cultures sans écriture disposeraient d'une mémoire « connective » qui serait fondée sur la réitération du rituel qui reproduit l'ordre immuable du cosmos. En revanche, parce qu'elles disposent d'un système de communication écrite, les sociétés «chaudes» auraient "intériorisé» (verinnerlicht) l'histoire et entretiendraient une mémoire historique, une véritable mémoire culturelle.

D'un côté donc la Bindungsgedächtnis, de l'autre la Bildungsgedächtnis : ce partage d'ordre à la fois structural et évolutif n'est pas sans évoquer la distinction romantique allemande entre Zivilisation et Kultur. En ce qui concerne en particulier les religions, ce partage s'inscrit une fois encore dans la vieille distinction entre les Stammeskulturen qui correspondent aux cultures tribales centrées sur la pratique rituelle et les "grandes civilisations » qui sont fondées sur une religion du livre et de la théologie qui en découle (Assmann 2000, p. 32-34 et 42-44). Dans cette perspective discriminante, l'écriture est donnée comme un instrument de libération assurant das freie Leben des Geistes; « la vie libre de l'esprit » est placée une fois encore sous l'autorité explicite de Hegel et, par la valorisation implicite de la Bildung, sous celle indirecte de Herder ... 
Inutile de dire que la Grèce classique échappe entièrement à ce nouvel avatar du Grand Partage, assorti de la valorisation implicite des "grandes civilisations». On a suffisamment dit ailleurs le rôle essentiel joué par les formes poétiques dans l'actualisation, la communication et la transmission des récits héroïques que nous identifions en tant que «mythes $»^{3}$. Fondement de la mémoire culturelle de chaque cité grecque, ces récits exemplaires n'existent que dans les formes chantées qui les actualisent en performances ritualisées dans des conditions de transmission institutionnelles. C'est ainsi que les hauts faits de la guerre de Troie ou de la guerre des sept contre Thèbes, pour ne pas parler des exploits instituants d'Érechthée ou de Thésée à Athènes, animent une mémoire culturelle partagée entre accomplissement rituel et tradition écrite grâce au système de notation alphabétique emprunté aux Phéniciens (voir par exemple Ford 2003). Sans doute n'est-ce donc pas un hasard si, entre religions de tradition rituelle et religions de tradition écrite (réduites pour l'essentiel aux trois monothéismes du Livre), entre Bindungsgedächtnis et Bildungsgedächtnis, la religion gréco-romaine n'est pas prise en considération par Assmann. Pourtant c'est bien l'étrangeté des manifestations de mémoire culturelle dans le polythéisme gréco-romain qui pourrait conduire à une attitude de critique et de rejet du paradigme moderne de la valorisation culturelle de l'écriture et des religions du Livre, dans une démarche du double écart.

\section{Sigmund Freud et la Kultur/« civilisation »}

Convient-il donc de jeter aux poubelles de l'histoire l'idée de civilisation? Et, pour rester dans la tradition germanophone, il y aurait-il désormais « malaise dans la civilisation »?

$\mathrm{Au}$ sens freudien du terme, la Kultur, traduite en français par "civilisation", semble devoir reposer sur trois piliers : premièrement les moyens techniques (les outils, les instruments) que l'homme se donne pour se protéger des forces naturelles; puis la transposition qu'opère l'être humain, par le travail de ses propres mains, du beau qu'il rencontre dans la nature; enfin l'exigence de l'hygiène requise autant par l'entretien que par celui de notre environnement que par notre propre corps, une exigence qui s'inscrirait contre le " penchant naturel » de l'homme à la négligence et au dérèglement. C'est donc dans Malaise dans la civilisation que Sigmund Freud tire de cette définition des bases de la civilisation la conclusion suivante : « La beauté, l'ordre et la propreté occupent à l'évidence une place particulière parmi les exigences de la civilisation " (Freud 2010, p. 89). Les trois fondements de la civilisation nous font donc passer de l'utilité des instruments techniques inventés par l'homme à la fabrication esthétique de la beauté pour s'accomplir, par le refoulement organique, dans l'hygiène d'un ordre. Or ce sont ces trois paramètres qui conduisent au trait le plus fondamental: la pratique des «activités psychiques supérieures» que sont les réalisations intellectuelles, scientifiques et artistiques ; parmi ces réalisations il faut compter en particulier la production d'idées par les systèmes religieux, les spéculations philosophiques ou les idéaux moraux de perfectionnement de l'homme et de l'humanité.

17 Mais dans la description freudienne, l'un des traits le plus prégnants de la Kultur/ civilisation réside dans le mode de régulation des rapports humains : "les relations sociales qui lient les hommes en tant qu'aides, voisins, objets sexuels, membres d'une famille ou d'un État» (Freud 2010, p. 91). Ces relations sociales sont essentielles à la civilisation puisque "la vie collective des hommes n'est rendue possible que par l'existence d'une majorité plus puissante que l'individu et solidaire contre chacun. Le 
pouvoir de la société s'oppose alors en tant que "droit" au pouvoir réprouvé de l'individu, la "force brutale" " (Freud 2010, p. 92). Règne du nómos sur la phúsis ? Socialement partagés, l'ordre, la règle, le droit seraient-ils capables de transformer et de maîtriser les "pulsions humaines», en particulier la pulsion de mort? La sublimation des pulsions, sinon le renoncement pulsionnel par le refoulement seraient alors essentiels au développement de la culture.

Néanmoins la perspective psychanalytique développée par Freud fait de l'individu l'entité qui précède la société. Dans cette mesure, la contradiction sociologique que l'on verra entre l'individualisme méthodologique, qui fait de l'individu l'indivis premier, et le holisme méthodologique, qui valorise le collectif des faits sociaux et les contraintes exercées par la société, ne reçoit pas de solution, sinon par l'intermédiaire d'une analogie. De même que l'individu est animé par le "ça » (partagé entre pulsions de vie et pulsions de mort, entre Éros et Thanatos), de même que l'individu réprime une libido et une agressivité qui, refoulée, est transformée en «surmoi » sous forme de culpabilité, de même la civilisation se doterait-elle d'un surmoi sous la forme d'une éthique; cette morale serait fondée, par exemple, sur un commandement tel que «tu aimeras ton prochain comme toi-même ». Quant au ça de la Kultur - et c'est là le problème - Freud nous renvoie sans précision au ça de l'individu ; un individu marqué par ce qui serait une tendance constitutionnelle des hommes, celle de s'agresser les uns les autres: « $\mathrm{Si}$ l'évolution de la civilisation ressemble autant à celle de l'individu et use des mêmes moyens, n'est-on pas en droit de diagnostiquer que bien des civilisations [...] sont devenues "névrosées" sous l'influence des tendances civilisatrices? (Freud 2010, p. 169). Conclusion face à l'agressivité constitutionnelle des êtres humains : "il reste à espérer que la "deuxième force céleste", l'Éros éternel, fasse un effort pour s'imposer dans sa lutte contre son adversaire tout aussi immortel. Mais qui peut prévoir l'issue de ce combat?»(Freud 2010, p. 171).

\section{Entre culture et individu : anthropopoiésis et écopoiésis}

C'est dire qu'au-delà d'un surmoi constitué en éthique, le ça civilisationnel nous réfère en définitive au conflit individuel entre Thanatos et Éros, au combat complémentaire entre pulsions de mort et volonté de destruction d'une part et pulsions sexuelles et exigence d'auto-conservation d'autre part, dans l'éternelle répétition (Freud 1927, p. 56-774). Le concept freudien de civilisation nous renvoie donc à l'individu et à son psychisme, à cet individu qu'en Europe occidentale on a constitué en sujet: un sujet qui, au siècle des Lumières, devient un être de raison, constitué en droit, un sujet libre de penser et libre d'agir comme il le veut ; un sujet dont les libertés sont fondées sur un certain nombre de droits, également partagés ; mais aussi un sujet pourvu d'une intériorité et d'une intimité singulières, capable de dialoguer avec lui-même; par conséquent un sujet réflexif qui va devenir le sujet philosophique qui se reconnait en tant que tel dans la mesure où il peut affirmer « je pense donc je suis »; ou encore un sujet pratique, un sujet agent engagé dans le monde extérieur selon une volonté et selon des motivations qui lui seraient propres; enfin, plus récemment, un sujet-individu capable de développer ses capacités propres et soucieux de réaliser son " self » par la performance dans la compétition et la concurrence avec les autres. Mais Freud est toujours là pour nous rappeler qu'entre le «ça » et le "surmoi» le sujet occidental est aussi sub-jectum, soumis à des contraintes qui le 
dépassent. Des contraintes d'ordre psychique sans doute, mais aussi des contraintes qui découlent de son indispensable insertion dans un réseau social et dans un environnement particuliers.

C'est ici qu'il convient de rappeler les termes du débat sociologique quant à savoir qui, de l'individu ou de la société, est premier par rapport à l'autre. Pour Émile Durkheim la société ne saurait être considérée comme la somme des individus qui la composent, des individus que l'on considérerait comme des entités premières, dans leurs différents états de conscience. Constituant un système qui a son autonomie et sa logique, la société impose aux individus des «manières d'agir et de penser ». Ainsi le social et le collectif ne peuvent être envisagés comme les simples résultantes des actions et des pratiques des individus : l' « individualisme méthodologique » doit être réfuté. Néanmoins la réduction qu'a opérée en contraste le « holisme méthodologique » en renvoyant les parties au tout a induit les sociologues à adopter une position de «relationalisme méthodologique»: société et individus sont à comprendre dans des relations qui correspondent à des rapports de réciprocité. Entre les individus-atomes comme êtres indivis et l'ensemble social auquel ils sont nécessairement intégrés, il y a interdépendance, interaction; il y a «co-construction» des uns par l'autre, et vice versa (voir Corcuff 2007, p.11-20 et 86-107).

21 Or, comme l'a fort bien appréhendé Freud, qui dit société dit « civilisation » et culture, qui dit société dit un ensemble de pratiques productives et symboliques, techniques et esthétiquement réglées, un ensemble animé sinon par une série d'idéaux, du moins par des représentations fortes, des récits fondateurs et des valeurs partagées qui, en différentes communautés, modèlent collectivement et singulièrement les actions et les pratiques des hommes tout en les légitimant. La culture donc qui peut être également une culture de la violence, de la discrimination et de la domination. Ainsi par le biais de pratiques d'ordre technique et symbolique où l'activité de la communication verbale joue un rôle essentiel, l'homme se constitue lui-même, pratiquement et symboliquement, dans son identité psychique, affective et réfléchie en interaction autant avec différents réseaux d'appartenance sociale et culturelle qu'avec un environnement singulier.

À partir d'une incomplétude aussi bien physique qu'intellectuelle déjà reconnue par Herder, à partir de ce qui est désormais identifié comme une plasticité neuronale exceptionnelle, l'être humain se construit comme sujet et comme individu en interaction obligée avec les différents cercles communautaires qui, en des termes freudiens que l'on retiendra volontiers, contribuent au façonnement de son ça et à la constitution de son surmoi: interaction avec les différentes valeurs, conventions, représentations et traditions qu'à son tour il adopte; interaction également avec un milieu qu'il contribue en retour à façonner quand il ne le détruit pas. En effet, en tant que représentations partagées et pratiques, ces "habitus" (pour reprendre le concept opportunément développé par Pierre Bourdieu) d'ordre culturel engagent l'individu à l'action sur l'environnement social, culturel et écologique qui a par ailleurs contribué à sa "fabrication ». À saisir par les deux concepts opératoires conjugués d'« anthropopoiésis » et $d^{\prime}$ " écopoiésis $\|^{5}$, ce processus se poursuit pendant toute sa carrière d'être humain mortel. L'individu se fabrique à la fois par les autres, pour les autres et contre les autres, dans des formes d'action et des formes de discours culturellement marquées et socialement acceptées (ou au contraire marginalisées).

D'un point de vue anthropologique, l'identité complexe de chaque homme repose donc sur une dialectique subtile entre d'une part la nécessaire fabrication sociale et culturelle 
de l'être humain par ses proches dans différents réseaux de sociabilité, et d'autre part le développement de la singularité de l'individu, dans son identité physique et psychique, dans son identité pratique et intellectuelle, dans son identité affective et morale. Donc sans civilisation, pas l'individu émancipé en personne avec sa réflexivité critique, attentive à celles et ceux qui contribuent à sa construction anthropopoiétique et écopoiétique, et à son constant maintien, physique et psychique, social et culturel.

Le cas échéant, l'émancipation pourra s'opérer avec l'aide des Grecs ou des Romains, mais dans une perspective d'anthropologie doublement critique. On plaidera donc pour une anthropologie (en l'occurrence historique) fondée sur un constant effort de «traduction transculturelle» (voir Calame 2002): retour aux pratiques politiques, sociales et religieuses, et surtout aux pratiques discursives propres aux Anciens, au plus près des termes et de leur signification « indigènes ", mais pour s'en distancer et nourrir ainsi la réflexion critique sur ce que nous en faisons dans un paradigme civilisationnel bien différent. Paradoxalement marqué par un individualisme concurrentiel dans des systèmes sociaux hyperconnectés et surinformés, le paradigme de la civilisation contradictoire du selfie et du réseau immédiat ne peut que bénéficier de la confrontation, par exemple, avec des formes de poésie chantée et collective, en performances ritualisées pour la construction anthropopoiétique d'individualités émancipées, femmes et hommes.

\section{BIBLIOGRAPHIE}

Affergan F., Borutti S., Calame C., Fabietti U., Kilani M., Remotti F. (2003), Figures de l'humain. Les représentations de l'anthropologie, Paris.

Andurand A. (2013a), "Friedrich August Wolf, "héros éponyme" de la Science de l'Antiquité », Anabases 17, p. 23-229.

- (2013b), Le mythe grec allemand. Histoire d'une affinité élective, Rennes.

Assmann J. (1992), Das kulturelle Gedächtnis : Schrift, Erinnerung und politische Identität in frühen Hochkulturen, Munich ; trad. angl. : 2011, Cambridge.

- (2000), Religion und Kulturelles Gedächtnis. Zehn Studien, Munich ; trad. angl. : 2005, Stanford.

Calame C. (2002), « Interprétation et traduction des cultures. Les catégories de la pensée et du discours anthropologique », L'Homme 163, p. 51-78.

- (2014), « From Cultural Memory to Poetic Memory: Ancient Greek Practices of History Beyond the "Great Divide" ", Fudan Journal of the Humanities and the Social Sciences 7-4, p. 639-652.

- (2015a), Avenir de la planète et urgence climatique. Au-delà de l'opposition nature/culture, Fécamp.

- (2015b), Qu'est-ce que la Mythologie grecque ?, Paris.

Cozzo A. (2011), «F. A. Wolf, la scienza dell'Antichità e noi : come possiamo uscire dal XIX secolo?", Mètis N. S. 9, p. 339-364.

Corcuff Ph. (2007), Les nouvelles sociologies, Paris (2 éd.).

Cuche D. (1996), La notion de culture dans les sciences sociales, Paris. 
De Luca M. et Ferry L. (2016), Thésée et le Minotaure, Paris.

Durkheim E. et Mauss M. (1913), « Note sur la notion de civilisation », Année sociologique 12, p. 46-50.

Ford A. (2003), « From Letters to Literature: Reading the "Song Culture" of Classical Greece », dans Yunis H. éd., Written Texts and the Rise of Literate Culture in Ancient Greece, Cambridge, p. 15-37. Freud S. (1927), Au-delà du principe de plaisir, dans Essais de psychanalyse, Paris, p. 7-148 ; éd. or. : Jenseits des Lustprinzips, Leipzig-Vienne-Zurich, 1920.

- (2010) Malaise dans la civilisation, Paris ; éd. or : Das Unbehagen in der Kultur, Vienne, 1930.

Goody J. (1977), The Domestication of the Savage Mind, Cambridge.

Herder J. W. (1784-1791), Ideen zur Geschichte und Philosophie der Menschheit, Riga.

Kilani M. (2009), Anthropologie. Du local au global, Paris ( $4^{\mathrm{e}}$ éd.).

Tylor E. B. (1871), Primitive Culture. Researches into the development of mythology, philosophy, religion, language, art, and custom, Londres.

Wolf F. A. (1807), « Darstellung der Althertums-Wissenschaft nach Begriff, Umfang, Zweck und Werth », Museum der Alterthums-Wissenschaft 1, p. 10-142.

\section{NOTES}

1. Voir De Luca et Ferry 2015, brièvement signalé par Antoine Duplan dans Le Temps, $\mathrm{n}^{\circ}$ 966, du 26 novembre 2016.

2. Cette dédicace a fait l'objet d'une traduction en français dans la contribution d'Andurand 2013a, p. 237-239. Dans l'ouvrage de 2013b, p.37-99, Andurand retrace l'histoire du lien de filiation établi entre Grèce classique et Allemagne romantique dès Johann Joachim Winckelmann et Wilhelm von Humboldt.

3. Voir par exemple Calame 2015b, p. 23-35 et 77-107.

4. On trouvera d'autres références à ce propos dans les bonnes pages que Laurie Laufer consacre à cette dialectique entre excès pulsionnels dans sa préface à la nouvelle traduction de Malaise dans la civilisation (Freud 2010, p. 22-35).

5. On verra à ce propos les différentes contributions publiées dans Affergan et al. 2003 ainsi que Calame 2015a, p. 71-110.

\section{RÉSUMÉS}

En énonçant les principes fondateurs de l'Alterthumswissenschaft, F. A. Wolf opère une distinction entre la «civilité bourgeoise" (die bürgerliche Policirung) des nations de l'Orient et la «culture spirituelle» (die Geisteskultur) des Grecs et des Romains. Des définitions anthropologiques (E. B. Tylor) ou sociologiques (E. Durkheim et M. Mauss) de la culture ou de la civilisation permettront d'échapper à la hiérarchisation entre les cultures. On trouvera en particulier dans la notion de 
civilisation (Kultur) telle qu'elle est développée par S. Freud des premières réponses à la question de la relation entre l'individu avec son psychisme et une société profondément marquée par sa culture. Et du côté de l'anthropologie, ce sera l'idée de la fabrication sociale et culturelle de l'homme en interaction avec son environnement qui invitera à revisiter les définitions de la civilisation proposées par Durkheim et Mauss ainsi que par Freud, sans accorder de privilège à la culture gréco-romaine.

When he outlines the fundamental principles of Alterthumswissenschaft, F. A. Wolf distinguishes between "die bürgerliche Policirung " of eastern Nations, and "die Geisteskultur » of Greeks and Romans. Other anthropological (E. B. Tylor) or sociological (E. Durkheim et M. Mauss) definitions of the notions of culture and civilization prevent us from creating a hierarchy of cultures. The vision of civilization developed by Freud, establishes a relationship between the individual, his psychic individuality and a society deeply engaged in its own culture. In an anthropological perspective, the idea of the social making of men in relation to their background leads us to take another look at Durkheim, Mauss and Freud's definitions of civilization, without favouring Greek and Roman culture.

\section{INDEX}

Mots-clés : civilisation, Kultur, « Grand Partage », fabrication sociale de l'individu, anthropopoiesis

Keywords : civilization, Kultur, « Great Divide », social production of the individual, anthropopoiesis

\section{AUTEUR}

\section{CLAUDE CALAME}

EHESS, ANHIMA UMR8210 